\title{
Attitudes et perceptions des médecins d'un hôpital universitaire du Liban à l'égard de l'enseignement et de l'apprentissage des habiletés de communication
}

\section{Attitudes and perceptions of a medical community of a Lebanese university hospital toward communication skills teaching and learning}

\author{
Grace ABI RIzK ${ }^{1}$, Éliane EID KHOURY ${ }^{2}$ et Claudine NASR HAGE ${ }^{1}$
}

1 Médecin de famille, Département de médecine familiale, Faculté de médecine, Université Saint Joseph, Beyrouth, Liban

2 Résidente en médecine de famille au moment de la recherche; actuellement, médecin de famille, Beyrouth, Liban

Manuscrit reçu le 5 octobre 2007; commentaires éditoriaux formulés aux auteurs le 26 novembre 2008 ; accepté pour publication le 23 décembre 2008

\author{
Mots clés : \\ Techniques \\ de communication ; \\ communication \\ médicale ; \\ étudiants ; \\ relation médecin \\ patient ; \\ attitudes ; \\ perception
}

Keywords:

Communication

skills;

medical communication;
Résumé - Contexte : Bien que les organismes d'accréditation internationaux exigent l'inclusion d'un enseignement des techniques de communication médecin-patient dans le curriculum des étudiants de médecine, ce type d'enseignement reste déficient dans la majorité des facultés de médecine au Liban. Buts : Évaluer les attitudes des médecins de la faculté de médecine de l'université Saint Joseph (USJ) exerçant à l'hôpital Hôtel Dieu de France (HDF), envers la communication médicale et estimer leurs perceptions de l'enseignement actuel de ces techniques. Matériels et sujets : Un questionnaire auto-administré respectant l'anonymat a été envoyé à tous les médecins de l'université Saint Joseph pratiquant à l'hôpital HDF $(n=154)$. Résultats : Cent trente et un (131) médecins ont répondu au questionnaire (85\% de réponse). Les médecins avaient des attitudes très positives envers les techniques de communication avec un score moyen des attitudes de 52,9 sur un total de 60 et une perception plutôt négative de l'enseignement actuel des techniques de communication aux étudiants de médecine avec un score moyen des perceptions de 14,8 sur un total de 35. Plus de la moitié des médecins ont estimé que la performance des étudiants dans les différents domaines de la communication médicale était faible. Conclusion : L'attitude positive que les médecins de la faculté de médecine de l'USJ ont exprimée et leur perception négative de l'enseignement actuel concernant la communication médicale invitent à envisager l'instauration d'un programme d'enseignement des techniques de communication s'appuyant sur l'adhésion des médecins à ce programme.

Abstract - Background: Although many international accreditation groups recommend to teach doctor-patient communication skills to medical students, there is still a lack of teaching of these skills in most Lebanese medical schools. Purpose: To evaluate the attitudes of clinical faculty members of the University Saint Joseph (USJ) working at the hospital 


$\begin{array}{ll}\text { students; } & \text { Hôtel-Dieu de France (HDF) towards medical communication and to assess their perceptions } \\ \text { doctor-patient } & \text { on the current communication skills teaching. Methods: An anonymous self-administered } \\ \text { relationship; } & \text { questionnaire was sent to all clinical faculty members working at the HDF ( } n=154) \text {. } \\ \text { attitudes; } & \text { Results: A total of } 131 \text { faculty members responded (85\% of response). Faculty members } \\ \text { perception } & \text { had a great positive attitude towards communication skills with a mean score of } 52.9 \text { out of } \\ & \text { a maximum of } 60 \text {. Physicians' perceptions of current communication skills teaching to med- } \\ & \text { ical students were rather negative with a mean score of } 14.8 \text { out of a maximum of 35. More } \\ & \text { than } 50 \% \text { of respondents have rated poor students' performance in different fields of medical } \\ & \text { communication. Conclusion: The positive attitude expressed by faculty members working } \\ & \text { at the HDF along with their negative perceptions of the current teaching in regards of medical } \\ & \text { communication suggest to consider the implementation of a communication skills teaching } \\ & \text { program relying on the adhesion of faculty members to this program. }\end{array}$

\section{Introduction}

La formation initiale des médecins ne les prépare pas toujours à la gestion de la relation avec leurs patients, qui est pourtant au cœur de l'activité soignante quotidienne, quel que soit le motif de la consultation. Une bonne communication entre le médecin et le patient est capitale pour une prise en charge optimale des patients : elle facilite les prises de décision, augmente la satisfaction du patient et son adhésion à la prescription médicale et améliore le pronostic de la maladie ${ }^{[1]}$. Les aspects communicationnels et relationnels constituent une dimension essentielle de toutes les activités cliniques des professionnels de la santé, qu'il s'agisse du recueil des données pour élaborer un diagnostic, de l'information, de l'écoute et de l'accompagnement des patients et de leurs familles ou encore de la démarche d'éducation thérapeutique ${ }^{[2]}$.

Un déficit de communication est en cause dans la plupart des plaintes judiciaires déposées à l'encontre des professionnels de santé; celles-ci sont liées notamment à la perception par le patient d'une attitude hautaine du médecin, à la perception d'un échec lors de l'échange d'informations ou d'un manque d'attention vis-à-vis d'un patient de la part d'un médecin pressé, par exemple ${ }^{[3,4]}$.

Jusqu'à une date récente, on considérait volontiers la communication médicale comme un art qui s'apprenait de façon non formelle et implicite, dans le cadre de l'exercice de la profession, ou comme relevant de caractéristiques individuelles des praticiens. Plusieurs études aujourd'hui disponibles ont montré que les techniques de communication peuvent être apprises explicitement et donc qu'elles peuvent être enseignées, au bénéfice d'une amélioration de la relation médecinpatient $^{[5,6]}$. Dès lors, l'importance de cette aptitude clinique est reconnue par de multiples organisations professionnelles internationales. Certaines, comme l'«Accreditation Council for Graduate Medical Education » ${ }^{[7]}$ exigent même qu'un programme dédié à l'apprentissage des techniques de communication (TC) soit inclus dans le curriculum des études médicales. D'autres, comme l' «American Board of Medical Specialties », ont inclus les TC dans leurs critères de certification et recertification des spécialités médicales ${ }^{[8]}$. Le «Royal College of Physicians and Surgeons of Canada», a clairement exprimé dans son rapport CanMEDS $2000^{[9]}$ le besoin d'une meilleure éducation dans le domaine de la communication médicale et requiert actuellement que soient satisfaits des objectifs bien définis concernant l'apprentissage des techniques de communication et qu'une évaluation formelle des compétences des résidents dans ce domaine soit effectuée.

Au Liban, parmi les sept facultés de médecine, seules deux d'entre elles ont implanté un dispositif d'enseignement des TC. À l'université américaine de Beyrouth (American University of Beirut), il existe un cours offert aux étudiants de cinquième année de médecine, qui consiste à exposer les étudiants 
à une série de consultations médicales filmées, suivies de discussions sur les bonnes et les mauvaises techniques de communication. À la faculté de médecine de l'université Saint Joseph (USJ), le département de médecine de famille est le seul à offrir un programme global d'enseignement des TC reposant sur des objectifs bien définis et recourant à des ateliers de formation sur la communication médicale pour les résidents de la spécialité. Ces ateliers couvrent presque tous les aspects d'une consultation médicale (différentes parties de l'entrevue médicale, annonce d'une mauvaise nouvelle, gestion d'un patient difficile, ...).

L'attitude des médecins enseignants à l'égard de la problématique de l'enseignement et de l'apprentissage des TC est un facteur primordial à prendre en compte dans un milieu de formation médicale, notamment de formation initiale. Des études ont montré qu'une attitude positive vis-à-vis d'un concept était associée à des comportements plutôt positifs ${ }^{[10]}$. Plusieurs articles de la littérature en éducation médicale préconisent de mettre en œuvre des ateliers de formation sur ce thème à l'intention des médecins enseignants eux-mêmes, pour améliorer leurs attitudes et leurs performances à cet égard, avant d'instaurer un programme formel d'enseignement et d'apprentissage des TC dédié aux étudiants ${ }^{[11-13]}$.

Ainsi, en vue d'optimiser la planification d'un programme global, et structuré d'enseignement et d'apprentissage des TC aux étudiants de la faculté de médecine de l'USJ et d'impliquer les médecins dans cet enseignement, il était essentiel d'évaluer les attitudes des médecins de la faculté de médecine de l'USJ envers la communication médicale et d'estimer leurs perceptions de l'enseignement actuel de ces techniques.

\section{Méthodes}

Type d'étude et population

Il s'agit d'une étude descriptive visant les médecins enseignants de la faculté de médecine de l'USJ, travaillant à l'hôpital Hôtel Dieu de France (HDF), durant la période de l'étude (septembre-novembre 2005). Le choix de la population s'est basé sur le fait que l'hôpital HDF constitue le centre hospitalier universitaire de référence affilié à la faculté de médecine de l'USJ et que la majorité des médecins de l'hôpital ont des obligations académiques envers les étudiants et sont en contact permanent avec eux. Les médecins qui n'avaient pas de contact avec les étudiants en médecine (médecins du personnel, radiothérapeutes) ou avec les patients (anatomo-pathologistes) ont été exclus.

\section{Instrument d'évaluation}

Un questionnaire a été distribué à tous les médecins de l'USJ pratiquant la médecine clinique à l'hôpital HDF. Les questionnaires ont été déposés dans les boîtes postales des médecins ou chez leurs secrétaires. Un casier spécial a été créé, permettant aux médecins de déposer le questionnaire rempli tout en gardant l'anonymat. Les médecins ont reçu deux appels téléphoniques, deux et six semaines après la distribution du questionnaire, leur rappelant la nécessité de le compléter.

Le questionnaire utilisé dans notre étude a fait l'objet d'une publication originale en anglais par Langille et al. en 2001 (Attitudes Towards Medical Communication Scale) ${ }^{[14]}$. Il a été développé par une équipe de recherche à l'université de Dalhousie au Canada. Ce questionnaire a été traduit en français dans la mesure où le français est la langue officielle à l'USJ et à l'HDF. Trois médecins de famille pédagogues maîtrisant le français et l'anglais ont révisé la version française et un traducteur indépendant a traduit de nouveau le questionnaire du français à l'anglais de manière à vérifier que la traduction française ne dénaturait pas le questionnaire princeps et que, dès lors, on pouvait postuler que la version française en conservait les qualités psychométriques de validité et de fidélité. Le questionnaire comprenait quatre parties explorant successivement les caractéristiques socio-démographiques et professionnelles des médecins, leurs attitudes envers les 
techniques de communication, leurs perceptions de l'enseignement actuel de ces techniques à l'hôpital HDF et l'appréciation par les médecins des aptitudes des internes et des résidents dans différents domaines de la communication médicale. Les réponses aux questions évaluant les attitudes (12 questions) et les perceptions (7 questions) ont été recueillies à l'aide d'une échelle de Likert à cinq niveaux de réponse allant du désaccord complet à l'accord complet, avec un score maximal de 60 pour les attitudes et de 35 pour les perceptions. Certaines questions étaient formulées selon une tournure inversée (où le désaccord complet implique une attitude positive envers les techniques de communication) pour éviter le biais de réponse automatique. L'appréciation par les médecins enseignants des aptitudes des étudiants dans le champ des TC a abordé six domaines de la communication médicale avec une échelle de réponse à quatre niveaux (mauvais, moyen, bon et excellent) et une cinquième option «je ne suis pas sûr » pour éviter d'induire des réponses non précises.

\section{Analyse statistique}

L'analyse statistique a été faite à l'aide du logiciel Stata version 7. Toutes les variables catégoriques ont été présentées en fréquences et pourcentages. Les variables générées par l'utilisation des échelles de Likert sont intrinsèquement de nature ordinale ; de ce fait il est, en toute, rigueur, illicite d'utiliser les tests statistiques paramétriques pour interpréter les données numériques qui en sont issues. Cependant, en nous appuyant sur la perspective méthodologique développée récemment par Carifio et Perla $^{[15]}$, nous avons d'une part agrégé les variables ordinales individuelles pour calculer des scores présentés en recourant au calcul des moyennes et de déviations standard (DS); postulant en outre que la taille suffisamment importante de l'échantillon permettait de présumer une distribution normale des variables, nous avons appliqué des tests paramétriques pour leur traitement statistique. L'analyse bivariée des scores en fonction des caractéristiques démographiques et professionnelles des médecins a été réalisée par des tests de Student et d'analyse de variance (ANOVA). L'analyse multivariée a consisté en une régression linéaire multiple des scores sur les variables indépendantes représentant les caractéristiques des médecins. Le seuil de significativité $\alpha$ a été choisi à 0,05 .

\section{Résultats}

Le questionnaire a été envoyé à tous les médecins de l'HDF ( $n=154$ médecins). Cent trente et un (131) questionnaires ont été remplis et remis, soit un taux de réponse de l'ordre de $85 \%$. Un seul questionnaire, dont seules les caractéristiques démographiques et professionnelles avaient été remplies, a été éliminé. Les caractéristiques des médecins qui ont participé à cette étude sont illustrées dans le tableau I. La répartition des répondeurs était respectivement de $81 \%$ (105) pour le sexe masculin et de $19 \%$ (25) pour le sexe féminin. La moitié des médecins exerçait depuis 10 à 20 ans à la faculté de médecine et à l'HDF; $20 \%$ seulement appartenaient à des disciplines considérées comme étant très impliquées dans la communication médicale (médecine de famille, pédiatrie, gynécologie et psychiatrie ${ }^{[14]}$. Quatre vingt quatorze pour cent (94\%) des médecins donnaient des cours à des étudiants en médecine non encore diplômés et $86 \%$ enseignaient à des résidents à l'hôpital. Cinquante-neuf médecins ( $n=59$; $45 \%$ ) avaient déjà assisté à un atelier consacré aux TC et 53 (41\%) ont affirmé avoir reçu une formation dans ce domaine. Seuls $25 \%$ d'entre eux lisaient des journaux abordant le thème de la communication médicale.

Le score moyen des attitudes envers les TC a été de 52,85 pour un score maximal de 60 ( $\mathrm{DS}=0,384$ ) indiquant que la plupart des praticiens qui ont répondu au questionnaire avaient des attitudes très positives envers les TC. Les scores spécifiques pour chaque item sont rapportés dans le tableau II.

En analyse bivariée, l'appréciation de l'enseignement des TC comme étant extrêmement important était associée à un score élevé sur l'échelle des attitudes envers les TC $(p=0,02)$. La participation 
Tableau I. Caractéristiques sociodémographiques et professionnelles des médecins de la faculté de médecine de l'université Saint Joseph ayant participé à l'étude $(n=130)$.

\begin{tabular}{lll}
\hline Caractéristiques & $\boldsymbol{n}$ & $\boldsymbol{\%}$ \\
\hline Age $<45$ ans & 69 & 53 \\
\hline Sexe masculin & 105 & 81 \\
\hline Nombre d'années de pratique $>10$ ans & 86 & 67 \\
\hline Diplôme de médecine générale obtenu à l'USJ* & 113 & 87 \\
\hline Résidanat effectué à l'Hôtel Dieu (USJ) & 89 & 68 \\
\hline Enseignement $>5$ h par semaine & 78 & 60 \\
\hline Participation à plus de deux activités académiques (ARCsł ${ }^{*}$ conférences, staffs, & & \\
enseignement au lit du malade) & 105 & 81 \\
\hline Enseignement auprès d'étudiants en médecine non diplômés & 121 & 94 \\
\hline Enseignement auprès de résidents & 112 & 86 \\
\hline Participation à un atelier sur les TC $\ddagger$ & 59 & 45 \\
\hline Formation sur l'apprentissage des TC & 53 & 41 \\
\hline Lecture de journaux sur les TC & 32 & 25 \\
\hline Considère l'enseignement des TC comme très ou extrêmement important & 126 & 97 \\
\hline Considère l'enseignement des TC comme très ou extrêmement agréable & 108 & 83 \\
\hline$*$ USJ : Université Saint Joseph & $\dagger$ ARCs : apprentissage du raisonnement \\
\hline clinique $\ddagger$ TC : techniques de communication & & \\
\hline
\end{tabular}

à des séances d'apprentissage du raisonnement clinique était associée à un score élevé avec une valeur $p$ proche de la signification statistique $(p=0,06)$, tandis que l'âge, le sexe, le fait d'avoir participé à des ateliers sur les TC ou de lire des journaux traitant les différents aspects de la communication médicale n'étaient pas associés à des scores significativement plus élevés sur cette échelle.

Les perceptions des médecins concernant les programmes actuels d'enseignement des TC à la faculté de médecine de l'USJ ont été évaluées grâce à sept questions à cinq niveaux de réponses. Le score moyen a été de 14,77 pour un score maximal de 35 (DS = 0,316, IC $95 \%=14,14-15,39)$. Les réponses aux différentes questions sont illustrées dans le tableau III. La majorité des médecins
( $n=111 ; 86 \%$ ) ont considéré qu'un enseignement supplémentaire sur les TC était nécessaire dans le curriculum, que les résidents ne recevaient pas suffisamment d'entraînement pour répondre aux exigences de la communication dans la pratique médicale $(n=95 ; 73 \%)$ et que la grande part de ce que les étudiants apprenaient se faisait par «osmose », en se référant aux attitudes, aux modèles et aux connaissances des instructeurs $(n=115 ; 89 \%)$.

Dans l'analyse bivariée, six variables étaient significativement associées à des scores de perceptions plus bas. Ces variables sont les suivantes : appartenir à une discipline médicale spécialisée $(p=0,05)$, avoir eu son diplôme de médecine générale à l'USJ ( $p=0,01)$, enseigner plus de cinq heures par semaine $(p=0,01)$, enseigner aux 
Tableau II. Scores moyens des réponses concernant les items évaluant les attitudes des médecins participants envers les techniques de communication.

\begin{tabular}{|c|c|c|}
\hline Item & $n *$ & $\operatorname{Moy}(\mathbf{D S}) \dagger$ \\
\hline 1- C'est très important de clarifier le traitement au patient & 129 & $4,9(0,3) \div$ \\
\hline 2- S'assurer que le patient a compris est généralement non nécessaire & 129 & $4,2(1,4)$ \\
\hline \multicolumn{3}{|l|}{ 3- Bien que la solidarité soit un état désirable pour une équipe de santé, } \\
\hline il y a peu de choses qu'un membre puisse faire pour la promouvoir & 120 & $3,8(1,1)$ \\
\hline 4- Une bonne communication est une aptitude clinique très importante & 129 & $4,8(0,4)$ \\
\hline \multicolumn{3}{|l|}{ 5- Le médecin et les autres professionnels de santé doivent collaborer } \\
\hline d'une manière plus efficace & 124 & $4,7(0,5)$ \\
\hline \multicolumn{3}{|l|}{ 6- Ce n'est pas nécessaire de prendre en considération l'expérience du patient } \\
\hline dans la relation médecin-patient & 124 & $4,0(1,0)$ \\
\hline \multirow{2}{*}{\multicolumn{3}{|c|}{$\begin{array}{l}\text { 7- La prise en charge des problèmes émotionnels du patient ne relève pas } \\
\text { de la responsabilité du médecin mais des psychiatres, des psychologues }\end{array}$}} \\
\hline & & \\
\hline ou des assistants sociaux & 125 & $4,2(0,9)$ \\
\hline $\begin{array}{l}\text { 8- Procurer des informations au patient sur l'hygiène de vie est très important } \\
\text { dans la pratique médicale }\end{array}$ & \multicolumn{2}{|c|}{ 8- Procurer des informations au patient sur l'hygiène de vie est très important } \\
\hline \multicolumn{3}{|l|}{ 9- Traiter les problèmes émotionnels et psychosociaux des patients est absolument } \\
\hline 10- Une bonne communication médecin-patient améliore la prise en charge du patient & 129 & $4,9(0,3)$ \\
\hline \multicolumn{3}{|l|}{ 11- Les patients ne sont pas généralement affectés par les réactions non verbales } \\
\hline \multicolumn{3}{|l|}{ 12- Les médecins doivent être conscients du langage du corps et de l'utilisation } \\
\hline Score total & & $\begin{array}{l}52,85 \\
(0,384)\end{array}$ \\
\hline $\begin{array}{l}\text { * Nombre de médecins } † \text { Moy : moyenne; DS : déviation standard } ¥ \text { Réponses sel } \\
5 \text { niveaux (complètement d'accord, plutôt neutre, plutôt en désaccord, complètement en } \\
\text { réponses (2-3-6-7-11) avaient une tournure inversée où le désaccord complet contribuai }\end{array}$ & $\begin{array}{l}\text { n une } \\
\text { désace }\end{array}$ & $\begin{array}{l}\text { chelle Likert à } \\
\text { ord). Certaines } \\
\text { ore plus élevé. }\end{array}$ \\
\hline
\end{tabular}

étudiants au lit du malade ( $p=0,0008)$, enseigner aux étudiants non encore diplômés $(p=0,003)$ et considérer l'enseignement comme extrêmement agréable $(p=0,04)$. Ceci peut être résumé en énonçant qu'un spécialiste diplômé de l'USJ, enseignant plus de 5 heures par semaine, donnant des cours à des étudiants non encore diplômés, enseignant des étudiants au lit du malade et considérant l'enseignement comme une activité extrêmement agréable, avait une perception médiocre de l'enseignement des techniques de communication dans notre établissement. 
Tableau III. Perceptions des enseignants cliniciens interrogés à l'égard du programme de formation actuellement en vigueur concernant l'enseignement des techniques de communication $(N=130)$.

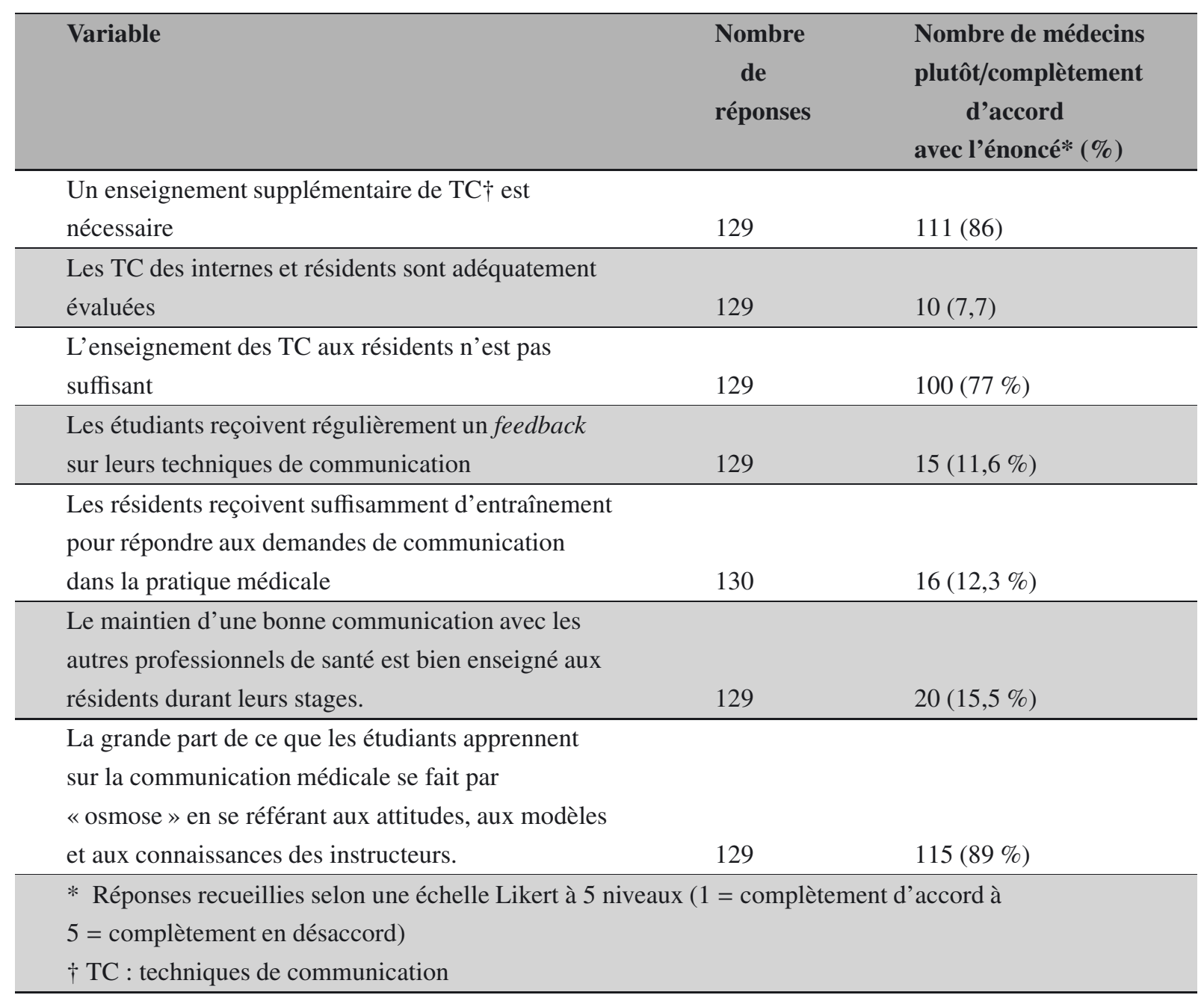

En régression linéaire, un nombre croissant d'activités académiques était inversement proportionnel au score des perceptions d'une façon statistiquement significative ( $p=0,04$, coefficient $\beta=-0,69$ ). Le score des attitudes était inversement corrélé au score des perceptions d'une façon significative ( $p=0,005$, coefficient de corrélation $\beta=0,25$ ). Les médecins ayant des attitudes très positives envers la problématique de l'enseignement des TC avaient une perception médiocre de l'enseignement actuel de ces techniques auprès des étudiants.
En dernier lieu, les médecins ont formulé une appréciation des aptitudes des internes (étudiants en sixième et septième année de médecine générale) et des résidents (étudiants diplômés en cours de spécialisation) au regard de six domaines de la communication médicale. Ils ont globalement considéré que tous les étudiants avaient un niveau pauvre de performance ; cependant les évaluations des résidents étaient meilleures que celles des internes dans les différents domaines. Les évaluations des résidents et des internes étaient statistiquement concordantes : les médecins qui ont évalué la performance 
Tableau IV. Appréciation par les médecins interrogés des performances des internes et résidents dans différents domaines impliquant des aptitudes dans le domaine de la communication médicale.

\begin{tabular}{llccc} 
& Internes & \multicolumn{2}{c}{ Résidents } \\
& & $\begin{array}{c}\text { Pourcentage } \\
\text { d'appréciation } \\
\text { négative } \\
\text { (mauvais + } \\
\text { moyen)* }\end{array}$ & $n$ & $\begin{array}{c}\text { Pourcentage } \\
\text { d'appréciation } \\
\text { négative } \\
\text { (mauvais + } \\
\text { moyen)* }\end{array}$ \\
Domaine & $n$ & $55 \%$ & 129 & $29 \%$ \\
\hline 1- Prise d'une bonne histoire médicale & 129 & $76 \%$ & 129 & $62 \%$ \\
\hline 2- Exploration du contexte familial & 129 & $78 \%$ & 129 & $60 \%$ \\
\hline 3- Conseils sur le style de vie du patient & 129 & $73 \%$ & 129 & $58 \%$ \\
\hline 4- Prise en charge des patients non compliants & 129 & $56 \%$ & 129 & $53 \%$ \\
\hline 5- Annonce d'une mauvaise nouvelle & 129 & $61 \%$ & 129 & $56 \%$ \\
\hline 6- Prise en charge des patients hostiles & 129 & & & \\
\hline * Réponses recueillies selon une échelle Likert à 4 niveaux (mauvais, moyen, bon, excellent) en \\
plus d'une option «je ne suis pas sûr »
\end{tabular}

des résidents comme pauvre ou moyenne dans un domaine donné avaient tendance à évaluer de façon identique celle des internes dans le même domaine. En somme, plus de la moitié des médecins ont estimé la performance des étudiants comme pauvre dans cinq des six domaines de la communication médicale. Les résultats sont illustrés dans le tableau IV.

\section{Discussion}

Notre étude est la première faite au Liban concernant l'attitude des médecins enseignants à l'égard des TC et leur perception de l'enseignement de ces techniques auprès des étudiants. Le taux de réponse est élevé (85\%), ce qui suggère que les répondants sont assez représentatifs de la population de l'étude. La grande majorité des médecins enseignants ont exprimé des attitudes très positives envers les TC mais ils sont insatisfaits de l'enseignement de ces techniques à la faculté de médecine de l'USJ. Ils considèrent aussi qu'un enseignement supplémentaire portant sur les TC est nécessaire dans le curriculum. Plus de la moitié des médecins estime que la performance des étudiants en matière de communication médicale est pauvre.

Les résultats de notre étude sont relativement conformes à ceux obtenus par Langille et al. à l'université de Dalhousie ${ }^{[14]}$. Les score des attitudes positives envers les TC sont assez proche (52,85 vs. 51,8 sur un total de 60). Dans notre étude, le point de vue que leur enseignement est une chose très ou extrêmement importante constituait la seule caractéristique de base déterminante d'un score plus élevé sur l'échelle des attitudes, ce qui, d'une certaine manière, relève d'une tautologie.

Dans l'étude de Langille et al., la participation préalable à un atelier sur les TC et l'appréciation de l'enseignement comme étant une activité « agréable » étaient également associées à des scores plus élevés. Nos données établissent par contre que, dans notre milieu, les médecins ayant reçu une formation à la communication médicale n'avaient pas d'attitudes plus positives que les autres médecins de l'étude $(p>0,05)$. Cette discordance avec les 
résultats de Langille et al. ${ }^{[14]}$ pourrait être expliquée par un biais de sélection : la majorité de nos participants avaient en effet une attitude très positive envers les $\mathrm{TC}$ avec une distribution très étroite des réponses à cet égard ; il est possible que les enseignants médecins plus intéressés par le sujet aient davantage participé à l'étude. Pour le confirmer, il conviendrait d'étudier les comportements explicites des médecins. Cependant, il est aussi possible que ces résultats soient limités par un biais social qui aurait poussé les médecins interrogés à répondre selon les normes jugées «désirables » ou «adéquates » et non pas selon la réalité de leurs attitudes ou opinions. Quoi qu'il en soit, les données disponibles préconisent de mettre en oeuvre des ateliers de formation visant à améliorer la performance des médecins dans le domaine de la communication indépendamment de leurs attitudes ${ }^{[11-13,16-18]}$.

Concernant les activités d'enseignement et d'apprentissage des TC telles qu'elles étaient implantées dans notre milieu académique au moment de l'étude, les médecins interrogés ont porté une appréciation critique en trouvant cet enseignement déficient. Ces résultats sont conformes avec ceux de l'étude de Langille et al. Dans leur étude comme dans la nôtre, une forte majorité de médecins (respectivement $85 \%$ et $89 \%$ ) admettent que l'apprentissage des TC n'est qu'informel et non explicite, par « osmose » à partir de l'observation des instructeursmodèles. Un tel constat est d'ailleurs la règle ${ }^{[19]}$, les études comparables à la nôtre ayant toujours été conduites préalablement à la réflexion visant à implanter un programme formel. Six variables étaient significativement associées à des scores bas sur l'échelle des perceptions. Trois de ces variables (enseigner plus de cinq heures par semaine, enseigner au lit du malade, donner des cours à des étudiants non diplômés) impliquent une participation active du médecin dans l'enseignement auprès des étudiants et des résidents; une quatrième variable (considérer que l'enseignement est une activité agréable) présage de dispositions favorables à l'engagement auprès des étudiants. Ainsi, le fait d'être activement engagé auprès des étudiants ou d'être disposé à le faire est associé à une plus grande insatisfaction à l'égard du défaut d'enseignement des TC.

Dans la dernière partie de notre questionnaire, les médecins ayant témoigné des attitudes très positives envers les TC et des opinions moins favorables à l'égard de l'enseignement actuel de ces techniques ont estimé que la performance des internes et des résidents dans cinq des six domaines de la communication médicale était faible. De telles données accréditent l'idée que ces médecins ont la conviction que le seul apprentissage par «osmose » en se référant aux modèles des instructeurs est insuffisant et qu'il est nécessaire de mettre en œuvre des activités d'enseignement et d'apprentissage formelles si l'on souhaite que les étudiants développent des aptitudes de communication au niveau qui est désormais attendu de la part de professionnels de santé.

Certaines limites de notre travail doivent être discutées. Celles liées à l'administration d'un questionnaire rédigé en français nous semblent mineures. Comme nous l'avons déjà mentionné, bien que la langue officielle au Liban soit l'arabe, le français représente la langue officielle à l'USJ et à l'hôpital HDF. Nous considérons dès lors que la probabilité d'une mauvaise compréhension du questionnaire par les participants est faible. Par ailleurs, nous avons respecté les procédures admises pour la traduction des questionnaires de recherche, ce qui permet de postuler que les qualités psychométriques du questionnaire princeps en anglais ont été préservées.

Nous considérons par ailleurs que le collectif des médecins ayant participé à l'étude est représentatif de la communauté des enseignants cliniciens de la faculté de médecine de l'USJ dans la mesure où l'hôpital HDF représente l'hôpital universitaire principal affilié a cette université. Cependant, le nombre de participants à cette étude a été limité par le nombre limite de la population-cible, celle des médecins de l'hôpital HDF. Ce petit nombre, eu égard au manque de puissance de l'étude qu'il induit, pourrait avoir occulté certaines associations entre les variables indépendantes et les scores mesurés. Au total, même si l'on peut admettre que 
d'autres études soient nécessaires pour autoriser une généralisation des résultats, il n'y a, à nos yeux, pas d'argument majeur de nature à suggérer que notre population d'étude soit très différente de celle des autres médecins-enseignants travaillant dans les centres hospitaliers universitaires au Liban.

L'attitude positive des médecins-instructeurs envers les TC devrait faciliter l'implantation d'activités formelles d'enseignement et d'apprentissage de ces techniques dans le curriculum des étudiants en médecine. Plusieurs implantations efficientes de programmes d'enseignement des TC ont été rapportées, par exemple au sein des facultés de médecine des universités de Maastricht ${ }^{[20]}$ et de Calgary ${ }^{[21]}$. Pour autant, il ne faut pas sous-estimer les difficultés d'une telle implantation. Plusieurs études ${ }^{[12,23,24]}$ soulignent l'importance d'une implication active de l'institution et de la communauté enseignante, l'intérêt d'une attitude positive des enseignants et l'utilité d'une formation adéquate des médecins-enseignants pour le succès d'un tel programme. La littérature disponible fournit peu d'informations à l'égard de ce qui serait le meilleur modèle d'implantation d'un tel programme; en revanche, il semble établi que le manque de soutien et de motivation de l'institution et des médecins-instructeurs constitue un obstacle ${ }^{[22]}$.

\section{Conclusion}

Cette première étude faite dans une faculté de médecine du Liban pour documenter l'attitude des médecins envers la problématique des TC et leurs perceptions de l'enseignement de ces techniques auprès des étudiants a clairement mis en évidence à la fois les insuffisances de la situation actuelle et une attitude très positive de la part des médecins enseignants. Ces résultats constituent un constat initial tout à fait essentiel à partir duquel le développement d'un programme institutionnel global dédié à l'enseignement et à l'apprentissage des TC, précédé d'une formation des cliniciens enseignants, va pouvoir être planifié tout au long des études médicales dans notre milieu institutionnel.

\section{Contributions}

Grace Abi Rizk a conçu le protocole de recherche et révisé le manuscrit. Éliane Khoury Eid a participé au recueil des données, à l'interprétation des résultatset à l'écriture du manuscrit. Claudine Nasr Hagea participé à l'écriture et à la révision du manuscrit.

\section{Références}

1. Stewart MA. Effective physician-patient communication and health outcomes - A review. Can Med Ass J 1995;152:1423-33.

2. Richard C, Lussier MT. La communication professionnelle en santé Montréal : Saint-Laurent (Québec) : Éditions du renouveau pédagogique - ERPI -, 2005.

3. Levinson W, Roter DL, Mullooly JP, Dull VT, Frankel RM. Physician-patient communication. The relationship with malpractice claims among primary care physicians and surgeons. JAMA 1997;277:553-9.

4. Kravitz RL, Callahan EJ, Paterniti D, Antonius D, Dunham M, Lewis CE. Prevalence and sources of patients' unmet expectations for care. Ann Int Med. 1996;125:730-7.

5. Kurtz SM, Silverman JD, Benson J, Draper J. Marrying content and process in clinical method teaching: Enhancing the Calgary-Cambridge guides. Acad Med. 2003;78:802-9

6. Yedidia MJ, Gillespie CC, Kachur E, Schwartz M, Ockene J, Chepaitis A et al. Effect of communications training on medical student performance. JAMA 2003;290:121012 .

7. American Council for Graduate Medical Education. Outcome project: toolbox of assessment methods. 2000 [On-line]. Disponible sur: http://www.acgme.org/ outcome/assess/toolbox. asp.

8. Kelley M. Components of clinical competence. 1999 [On-line]. Disponible sur : http://www . abms . org.

9. Frank JR (Ed). The CanMEDS 2005 physician competency framework. Better standards. Better physicians. Better care. Ottawa: The Royal College of Physicians and Surgeons of Canada, 2005. [On-line]. Disponible sur: http://crmcc.medical.org/canmeds/ CanMEDS2005/index.php 
10. Fazio R. Multiple processes by which attitudes guide behavior: The MODE model as an integrative framework. In: Zanna (Ed.) Advances in Experimental Social Psychology. San Diego(CA): Academic Press, 1990.

11. Lang F, Everett K, Mc Gowen R, Bennard B. Faculty Development in Communication Skills Instruction: Insights from a Longitudinal Program with "Real-time Feedback". Acad Med 2000;75:1222-8.

12. Egnew T, Mauksch L, Greer T. Integrating communication training into a required family medicine clerkship. Acad Med 2004;79:737-43.

13. Simpson M, Buckman R, Stewart M, Maguire P, Lipkin M, Novack D et al. Doctor-patient communication: the Toronto consensus statement. BMJ 1991;303:1385-7.

14. Langille D, Kaufman D, Laidlaw T, Sargeant J, MacLeod H. Faculty attitudes towards medical communication and their perceptions of students' communication skills training at Dalhousie University. Med Educ 2001; 35:548-54.

15. Carifio J, Perla R. Resolving the 50-year debate around using and misusing the Likert scales. Med Educ 2008;42:1150-2.

16. Duncan A, Multari A. Curriculum in physician-patient communication skills for new faculty. Acad Med 2002; 77:462.

17. Parker D. A workshop on mentoring across gender and culture lines. Acad Med 2002;77:461.
18. Bulik R, Frye A. A workshop on teaching beliefs. Acad Med. 2002;77:456.

19. Kurtz S, Laidlaw T, Makoul G, Schnabl G. Medical education initiatives in communication skills. Cancer Prev Control 1999;3:37-45.

20. van Dalen J, Zuidweg J, Collet J. The curriculum of communication skills teaching at Maastrict Medical School. Med Educ 1989;23:55-61.

21. Kurtz SM, Heaton CJ. Teaching and assessing information giving skills in communication curriculum. In: A Rothman, R Cohen, eds. Proceedings: Sixth Ottawa Conference on Medical Education. Toronto: University of Toronto, 1994.

22. Kurtz SM, Silverman J. The Calgary-Cambridge referenced observation guides: an aid to defining the curriculum and organizing the teaching in communication training programmes. Med Educ 1996;30:83-9.

23. Losh D, Mauksch L. Teaching inpatient communication skills to medical students: an innovative strategy. Acad Med 2005;80:118-24.

24. Rosenbaum M, Ferguson K. Teaching medical students and residents skills for delivering bad news: A review of strategies. Acad Med 2004;79:107-17.

Correspondance et offprints : Dr Grace Abi Rizk, Service de médecine familiale, Hôtel Dieu de France, Beyrouth, Liban. 